\title{
Information and measurement system of weapon stabilization parameters based on precision piezoelectric sensitive element
}

\author{
Andrii Tkachuk ${ }^{1, *}$, Olena Bezvesilna ${ }^{2}$, Oleksandr Dobrzhanskyi ${ }^{1}$, Anna Ostapchuk ${ }^{3}$, and Mykola Horodyskyi ${ }^{4}$ \\ ${ }^{1}$ Zhytomyr Polytechnic State University, Department of Automation and Computer-Integrated Technologies, Zhytomyr, Ukraine \\ ${ }^{2}$ National Technical University of Ukraine "Igor Sikorsky Kyiv Polytechnic Institute", Instrumentation Department, Kyiv, Ukraine \\ ${ }^{3}$ Zhytomyr Polytechnic State University, Theoretic and Applied Linguistics Department, Zhytomyr, Ukraine \\ ${ }^{4}$ Zhytomyr Polytechnic State University, Audit and Accounting Department, Zhytomyr, Ukraine
}

\begin{abstract}
The structure and principle of information and measurement system performance of weapon stabilization are described. Established, that the classical stabilization system consists of a stabilizing platform of the sensitive element (or block of sensitive elements), defining the absolute angle of platform $\alpha$ rotation, the sensing element output signal amplifier, engines and tachometers. The classical system of indirect stabilization is reviewed, its mathematical description is made. A new precision sensitive element for the information and measurement system of weapon stabilization is suggested, the principle of its performance is introduced. Constructive methods of measurement accuracy improvement in the new sensitive element are analyzed. A simulation of the new sensitive element performance influenced by the external disturbances is carried out.
\end{abstract}

\section{Introduction}

The Sustainable Development Strategy "Ukraine 2030" defines the purpose, vectors of movement, roadmap, priority and indicators of adequate defense, socioeconomic, organizational, political and legal conditions of formation and development of Ukraine. The main purpose of the reform of the national security and defense is to improve the defense of the state, reforming the Armed Forces of Ukraine and other military formations of Ukraine in accordance with modern requirements and the experience gained during the counterterrorist operation and development of defense industry to maximize meet the needs of the army. The Armed Forces of Ukraine, priority should be given full renovation of structures, ranging from governments and ending with the staff list and units, as well as optimization of systems and standards support, the introduction of new models of weapons and military equipment, revision of tactics and strategies to meet the requirements of today.

One of the trends in the development of modern combat vehicles fire control systems is the development of a more effective weapon stabilizer with high precision parameters contributing to the increase of the military combat vehicle power and the survivability of the "crewmachine" system. In this regard, the analysis of the development of modern weapons stabilizers is an urgent task.

Relating to this, the analysis of the contemporary weapon stabilizers development is a relevant issue. Technically, the stabilizer is a set of sensors and a computing complex connected to the gun drive. Based on the sensor performance, the parameters of the platform displacement are determined and the gun actuator is provided with control commands compensating the deviation.

In the article, an automatic control system is considered as a system of stabilization ensuring the maintenance of certain angular tower orientation in relation to the coordinate system the axes of which in a certain way are oriented in space. This coordinate system is a reference one. Depending on the specific task, this may be, for example, an inertial coordinate system or a system the axes of which are directed vertically to the moving object.

\section{Literature review}

Today, the development of new information and measurement weapon stabilization complexes of its sensitive elements is performed by scientists of National Technical University of Ukraine "Igor Sikorsky Kyiv Polytechnic Institute", Zhytomyr Polytechnic State University in cooperation with the public joint-stock company "Scientific and Production Association "Kyiv Automation Plant" [1].

Therefore, the relevant scientific and technical issue today is to improve the accuracy of the weapon stabilization complex.

In [2], an analysis of the current armored vehicles condition in Ukraine is performed. The effectiveness of new weapon stabilization complexes development is explained.

In [3] the structure of the pointing and armor

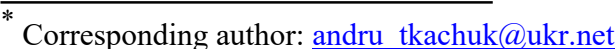


stabilization system of light armored vehicles with a neural network controller is described. However, the point of using a neural network approach to compensate the instrumental errors has not been considered.

In [4-5], it is proposed to use a coriolis vibrating gyroscope as a sensitive element of the stabilization system. The make-up and principle of the well-known navigation complex strike- and vibroprotection of the light armored vehicles system performance is analyzed. However, there are no suggestions to improve the system technical characteristics, mathematical model and errors of the system sensitive elements analysis.

In [6], schematic models were constructed and piezoelectric transducers with additional electrical oscillating circuits to be used as a sensitive element of a weapon stabilizer were simulated. The use of models like that allows the use of applications to evaluate the characteristics and prediction of piezoelectric converters parameters and performance modes. However, the results obtained cannot be used as evidence that a sensor of such a design is more accurate than its counterparts, since no two-channel measurement error compensation schemes are considered.

Article [7] describes the use of MEMS technologies to develop sensitive elements for measuring acceleration and vibration.

The purpose is to develop a new precision sensitive element of the information and measurement system of the weapon stabilization parameters.

\section{The structure of information and measurement system of weapon stabilization}

The oscillations of the mobile military equipment hull are random and never stop during its movement. The amplitudes of the angular oscillations have quite high frequencies. This leads to significant movement of the target mark relative to the target and does not allow the gun pointer to hold it even with the most advanced actuators.

The oscillations in the longitudinal plane changing the angle of the gun elevation, and angular oscillations in the horizontal plane, changing the angle of horizontal guidance, have the greatest influence on the firing accuracy. They lead to considerable dispersion of shells and bullets.

Transverse angular oscillations causing the gun to tilt have less impact, but grow with the firing distance.

First and foremost, these factors cause the raise in pointing errors, increasing by 10-30 times when firing from the move compared to the stationary fire.

The classical stabilization system consists of a stabilizing platform of the sensitive element (or block of sensitive elements), defining the absolute angle of platform $\alpha$ rotation, the sensing element output signal amplifier, engines and tachometers.

The block diagram of the classical indirect stabilization system is presented in [8-9] and is shown in Fig. 1, where SE is a sensitive element; A - amplifier; E engine; $\mathrm{R}$ - reducer; $\mathrm{P}$ is a stable platform; $W_{1}(p)$ and
$W_{2}(p)$ are the transfer functions of the amplifier units together with the correction means; $X(p)$ is the transfer function of the signal chain by the angle derivatives of the platform rotation $\alpha_{2}$ with respect to the oscillating object $\lim \underset{p \rightarrow 0}{X}=0 ; K(p)$ is the transfer function of the signal chain by the oscillation angle derivatives $\theta, \lim \underset{p \rightarrow 0}{K}=0$.

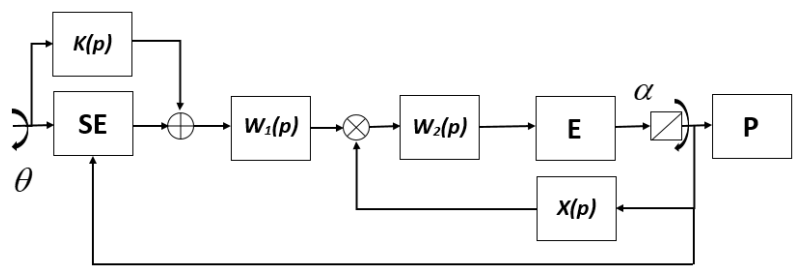

Fig. 1. Block diagram of the weapon indirect stabilization.

In [1] an example of a stabilization system that is recommended to be used as a part of aviation gravimetric systems is highlighted. In this system, the horizontal stabilized platform (GSP) has two linear accelerometers and actuators represented by special engines.

The stabilization system functions in the following way: the output signals of the linear accelerometers $f_{y}, f_{x}$ installed on a GSP oriented in a geographical coordinate system, the sensitivity axes, respectively forwarded to north and east, will have the form [8]:

$$
\begin{gathered}
f_{x}=-\left(2 \dot{r} \dot{\phi}_{c}+r \ddot{\phi}_{c}\right) \cos \chi+\left(\ddot{r}-r \dot{\phi}_{c}^{2}\right) \sin \chi- \\
2 r \omega_{E} \dot{\lambda} \cos \phi_{c} \sin \phi-r \dot{\lambda} \cos \phi_{c} \sin \phi+Q g ;
\end{gathered}
$$

$$
\begin{array}{r}
f_{y}=2 r \dot{\phi}_{c} \omega_{E} \sin \phi_{c}+2 r \phi_{c} \dot{\lambda} \sin \phi_{c}- \\
-2 \dot{r} \dot{\lambda} \cos \phi_{c}-r \ddot{\lambda} \cos \phi_{c}-2 \dot{r} \omega_{E} \cos \phi_{c}-v g,
\end{array}
$$

where $Q, v$ are the angles between the normals to the ellipsoid and geoid respectively in the meridional cross section and in the cross-sectional plane perpendicular to the meridian plane;

$\phi, \phi_{c}$ - respective geographical and geocentric latitude;

$\chi$-deviation from the vertical;

$\lambda$ is the longitude;

$g$ is the acceleration;

$r$ is the radius of the location;

$\omega_{E}$ is the Earth rotation speed.

The received signals are directed to the onboard computer where the control signal is emerging, then coming to the engines that further adjust the GSP to zero.

If the GSP is set exactly in the vertical position, the horizontal components of the gravity acceleration are equal to zero. Considering that $Q_{g}=-v g=0$, the components the onboard computer will compensate are the following:

$$
\begin{gathered}
f_{x} \Rightarrow 0=2 r \omega_{E} \dot{\lambda} \cos \phi_{c} \sin \phi-r \dot{\lambda} \cos \phi_{c} \sin \phi \\
f_{y} \Rightarrow 0=2 r \dot{\phi}_{c} \omega_{E} \sin \phi_{c}+2 r \phi_{c} \dot{\lambda} \sin \phi_{c}- \\
-2 \dot{r} \dot{\lambda} \cos \phi_{c}-2 \dot{r} \omega_{E} \cos \phi_{c} .
\end{gathered}
$$

Neglecting the components of the second order and taking the deviation from the vertical equal to zero, there we have: 


$$
\begin{gathered}
f_{x}=-r \ddot{\phi}_{c} ; \\
f_{y}=-2 r \ddot{\lambda} \cos \phi_{c} .
\end{gathered}
$$

If case each of the signals $f_{y}$ and $f_{x}$ is multiplied by $r^{-1}$, integrated and multiplied by $(-1)$, then at the output of the corresponding channels we get $\dot{\phi}$ and $\dot{\lambda} \cos \phi$. The signal $\dot{\phi}$ will be used to control the GSP with respect to the $x$ axis facing north, and the signal $\dot{\lambda} \cos \phi$ - to control the $y$ axis facing east.

In [10] both new high-precision piezoelectric and capacitive, string, gyroscopic sensitive elements are described. They can be used as parts of a stabilization system having their advantages and disadvantages. The use of the dual-channel method to eliminate the effect of instrumental errors on the accuracy of the conversion device is described.

Nowadays, the investigation of piezoelectric sensitive elements accuracy improvement is advanced. The new precision piezoelectric sensing element being more accurate than the already existing has been developed by the article authors.

\subsection{Signal conversion in piezoelectric sensing element}

The analysis of the signal conversion structural diagram in the piezoelectric element is suggested:

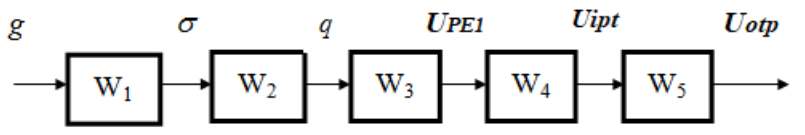

Fig. 2. Block diagram of direct signal conversion in piezoelectric sensing element.

Block $W_{1}$ corresponds to the conversion of acceleration $g$, acting on PE, into mechanical stress $\sigma$. This mechanical stress is numerically equal to the force $G$, per unit cross-sectional area of PE. If the mechanical stress is constant over the entire cross-sectional area PE $S$, then $\sigma=G / S$, therefore [11] (Fig. 2):

$$
W_{1}=\frac{\sigma}{G}=\frac{G}{S} \cdot \frac{1}{G}=\frac{1}{S}
$$

Block $W_{2}$ corresponds to the conversion of mechanical stress $\sigma$ into charge $q$ on PE electrodes:

$$
W_{2}=\frac{q}{\sigma}=\frac{d_{i j} \cdot \sigma \cdot S}{\sigma}=d_{i j} S
$$

where $d_{i j}$ - piezomodule.

Block $W_{3}$ corresponds to the conversion of charge $q$ into the voltage of $U_{P E}$ at $\mathrm{PE}$ electrodes. The transformation of this type is reflected in Fig. 3. diagram.

In accordance with the current $I$ scheme in Fig. 3 there can be written:

$$
I=\frac{d q}{d t}=\frac{U_{P E}}{R_{P E}}+C_{P E} \frac{d U_{P E}}{d t} .
$$

If we accept zero initial conditions, then the solution of the differential equation will the following:

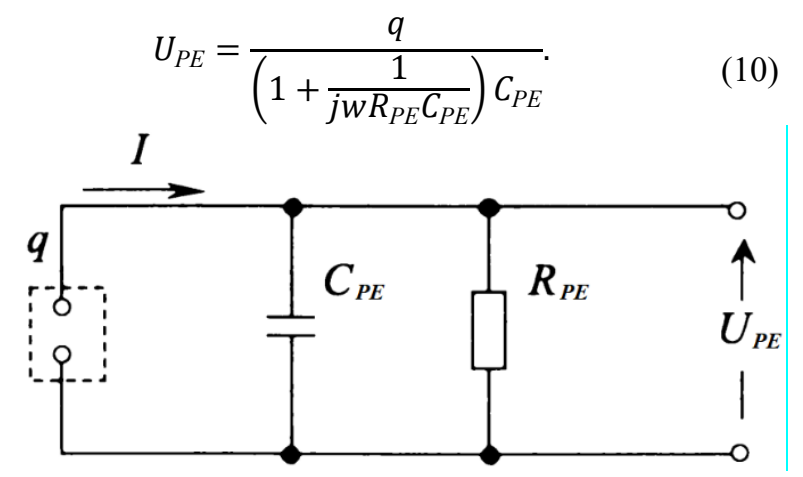

Fig. 3. Equivalent $\mathrm{W}_{3}$ link conversion scheme.

Hence the transfer function will be:

$W_{3}=\frac{U_{P E}}{q}=\frac{q}{q \cdot\left(1+\frac{1}{j w R_{P E} C_{P E}}\right) C_{P E}}=\frac{1}{\left(1+\frac{1}{j w R_{P E} C_{P E}}\right) C_{P E}}$.

However, given the large value of $R_{P E}$, it is possible to accept:

$$
W_{3}=\frac{1}{C_{P E}}
$$

Block $W_{4}$ describes the process taking place when the voltage on PE (transfer $U_{P E}$ in the circle $U_{i p t}$ ). Its transfer function corresponds to the transfer function of the modifying differential link, where $R_{B X}-$ input voltage amplifier resistance. Given the high PE resistance, the input of the amplifier is represented only by the resistance $R_{\text {ipt }}$.

$$
\begin{gathered}
U_{i p t}=\left(I_{1}+I_{2}\right) \cdot R_{i p t}, \\
I_{1}=C_{P E} \frac{d U_{C_{P E}}}{d t} \\
I_{2}=\frac{U_{C_{P E}}}{R_{P E}}
\end{gathered}
$$

In the result of substitution (14-15) in (13) we obtain:

$$
U_{i p t}=\left(C_{P E} \frac{d U_{C_{P E}}}{d t}+\frac{U_{C_{P E}}}{R_{P E}}\right) R_{i p t}
$$

According to Kirchhoff's law:

$$
U_{C_{P E}}=I_{2} R_{P E}=U_{P E}-U_{i p t} .
$$

Substituting (17) into (16) obtain:

$$
\begin{aligned}
& U_{i p t}=C_{P E} R_{i p t} \frac{d\left(U_{P E}-U_{i p t}\right)}{d t}+ \\
& +\frac{R_{i p t}}{R_{P E}}\left(U_{P E}-U_{i p t}\right) .
\end{aligned}
$$

For further calculations we introduce the coefficient:

$$
\varepsilon_{R}=\frac{R_{P E}+R_{i p t}}{R_{i p t}}
$$

Divide expression (18) by the coefficient $\varepsilon_{R}$ : 


$$
\begin{aligned}
& \frac{U_{i p t}}{\varepsilon_{R}}=\frac{C_{P E} R_{i p t}}{\varepsilon_{R}} \cdot \frac{d U_{P E}}{d t}-\frac{C_{P E} R_{i p t}}{\varepsilon_{R}} \cdot \frac{d U_{i p t}}{d t}+ \\
& +\frac{1}{\varepsilon_{R}} \frac{R_{i p t}}{R_{P E}} U_{P E}-\frac{1}{\varepsilon_{R}} \frac{R_{i p t}}{R_{P E}} U_{i p t} .
\end{aligned}
$$

Group the members of equation (20) and get rid of the denominator $R_{P E}$ :

$$
\begin{gathered}
\quad \frac{R_{P E} U_{i p t}}{\varepsilon_{R}}+\frac{R_{i p t}}{\varepsilon_{R}} U_{i p t}+\frac{C_{P E} R_{i p t} R_{P E}}{\varepsilon_{R}} \cdot \frac{d U_{i p t}}{d t}= \\
=\frac{C_{P E} R_{i p t} R_{P E}}{\varepsilon_{R}} \cdot \frac{d U_{P E}}{d t}+\frac{R_{i p t}}{\varepsilon_{R}} U_{P E}, \\
U_{i p t}+\frac{C_{P E} R_{P E}}{\varepsilon_{R}} \cdot \frac{d U_{i p t}}{d t}=\frac{1}{\varepsilon_{R}}\left(C_{P E} R_{P E} \cdot \frac{d U_{P E}}{d t}+U_{P E}\right) .
\end{gathered}
$$

Denote the time constant $\tau=R_{P E} C_{P E}$. In the result of the time constant by equation substitution (22) and the Laplace operator introduction, we obtain:

$$
\left(1+\frac{\tau}{\varepsilon_{R}} p\right) U_{i p t}=\frac{1}{\varepsilon_{R}}(1+\tau p) U_{P E} .
$$

The $W_{4}$ transfer function will be:

$$
W_{4}(p)=\frac{U_{i p t}}{U_{P E}}=\frac{1}{\varepsilon_{R}} \frac{1+\tau p}{1+\frac{\tau}{\varepsilon_{R}} p} .
$$

The $W_{5}$ corresponds to the voltage amplifier transmission characteristic [11], behaving as a first order inertial link:

$$
W_{5}(p)=\frac{K_{V A}}{1+p \tau_{V A}},
$$

where $K_{V A}$ - coefficient of the operational amplifier gain; $\tau_{V A}-$ time constant.

However, given the operation of the converter mainly in the narrow frequency range, the transmission characteristic can only be equated to the gain:

$$
W_{5}=K_{V A} .
$$
be:

Therefore, the transfer function of the signal in PE will

$$
\begin{aligned}
& W(p)=W_{1} W_{2} W_{3}(p) W_{4}(p) W_{5}= \\
& =d_{i j} \cdot \frac{1}{C_{P E}} \cdot \frac{1}{\varepsilon_{R}} \frac{1+\tau p}{1+\frac{\tau}{\varepsilon_{R}} p} \cdot K_{A V}
\end{aligned}
$$

However, PE has a tendency to discharge, so a negative feedback loop must be added to the direct signal conversion scheme.

\subsection{Three-coordinate piezoelectric sensitive element}

Increasing the measurement accuracy of the new precision sensitive element of the stabilization system is ensured by the fact that for each of the measurement axis $O z, O x$ and $O y$ on the GSP a sensitive element is installed. The sensitive element is made of two channels, each of which has one piezoelectric element being identical. The inertial masses are attached to the piezoelements piezoplates bottom of one channel and to the top of the piezoelements piezo-plates of the second channel. The first channel piezoelectric element of each sensitive element operates on the basis of extensional strain, and the second channel piezoelectric element is based on the compression (Fig. 4).

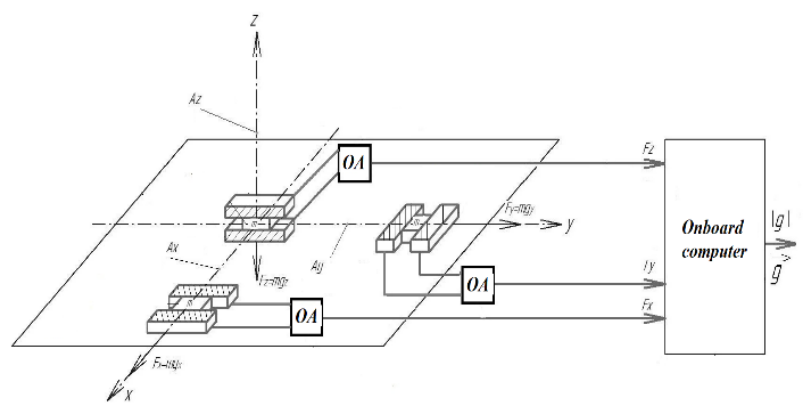

Fig. 4. Block diagram of the precision piezoelectric sensitive element.

The three-coordinate sensitive element operates as follows: the piezoelectric elements of both channels of the three sensitive elements $A z, A x, A y$, located along the axes $O z, O x$, and $O y$, are respectively accelerated. Also, the vertical acceleration of the aircraft and instrumental errors, in the result of residual non-identity of the same piezoelectric and mass structures, as well as in the result of changes in temperature, humidity and ambient pressure, affect each of the measurement axes.

If we use the algorithm of the two-channel measurement scheme performance for the two-channel piezoelectric element $A z, A x, A y$, we will receive as the on-board computer inputs 13 three signals having double values $2 g_{z}, 2 g_{x}, 2 g_{y}$ with no vertical plane acceleration and instrumental errors value resulting in the influence of residual non-identity of identical piezoplates structures and masses, the influence of temperature change, humidity and ambient pressure. These signals are added to and amplified in the performance amplifiers OP10, OP11, OP12 and are fed to the on-board computer 13, determining:

- full acceleration vector:

$$
\vec{g}=\overrightarrow{g_{x}}+\overrightarrow{g_{y}}+\overrightarrow{g_{z}}
$$

- full acceleration module:

$$
\begin{aligned}
& \quad|g|=\sqrt{\left(2 k m g_{x}\right)^{2}+\left(2 k m g_{y}\right)^{2}+\left(2 k m g_{z}\right)^{2}}= \\
& =2 k m \sqrt{g_{x}^{2}+g_{y}^{2}+g_{z}^{2}},(28)
\end{aligned}
$$

where $m$ is the inertial masses 7,8,9 in each piezoelectric element;

$k$ - piezoelectric constant being the same for all piezoelectric elements because of their identity.

The onboard computer takes control of the required calculations resulting in sending a control signal to the GSP engines. Also, the onboard computer output will get the full vector value and the full acceleration module, having no instrumental errors effect in the result of the 
residual non-identity influence of the same piezoelectric and mass structures, the influence of temperature changes, humidity and ambient pressure.

\subsection{Using dual-channel method}

The measurement accuracy improvement is possible due to use of the dual-channel method. The method is explained by the drawing, depicting a structural diagram of a piezoelectric sensitive element (Fig. 5) [10].

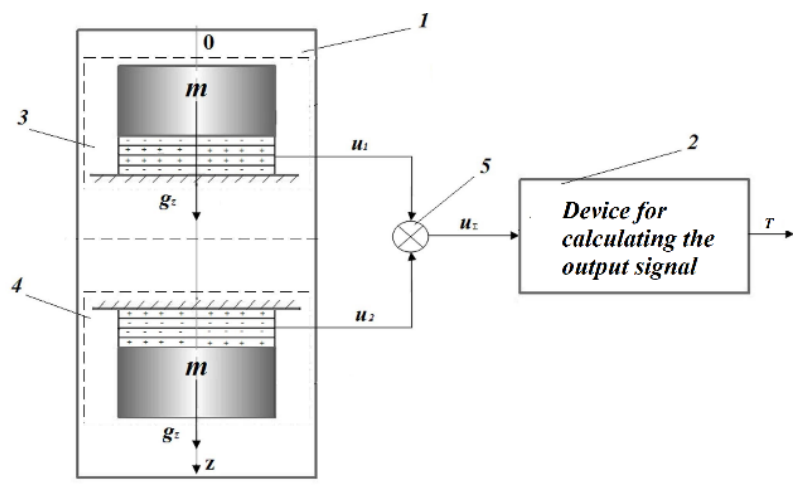

Fig. 5. Block diagram of the two-channel piezoelectric sensitive element.

The sensitive element 1 is made up of two channels, with the each one having a piezoelectric element. The piezoelectric elements of both channels are identical and are represented by piezo-plates and inertia mass, fixed on top of each other. The piezoelectric element 3 of one channel the piezo-plates of which are placed downwards, and the piezoelectric element 4 of the other channel, the piezo-plates of which are located upwards. The piezoplates outputs of both channels are connected to the inputs of the adder 5 , the output of which is connected to the input of the output signal calculation device 2 .

The piezoelectric elements of both channels are affected by acceleration $g$, vertical acceleration $\Delta \ddot{z}$ and instrumental errors $\Delta i$ resulted in the effects of residual non-identity of the same piezoelectric and mass structures, the influence of temperature changes, humidity and ambient pressure. If all these effects are exposed on the $O z$ axis of the new sensitive element considering that the piezoelement 3 of one channel compresses and the piezoelement 4 of the other channel stretches, we obtain [1]:

$u_{1}=k(m g+m \Delta \ddot{z}+\Delta i), u_{2}=k(m g-m \Delta \ddot{z}-\Delta i)$,

where $u_{1}$ - the output electric signal of one piezoelement channel; $u_{2}$ is the output electric signal of the other piezoelement channel; $m$ is the inertia mass weight in each of the channel; $k$ is the piezoelectric constant.

The output electric signals $u_{1}$ and $u_{2}$ of both piezoplates channels are summed up in the adder 5:

$$
u_{\Sigma}=u_{1}+u_{2}=2 k m g
$$

where $u_{\Sigma}$ is output adder 5 signal.

The output adder 5 signal is fed to the output calculating device where it is calculated over a certain period of time. In the result, we obtain the output signal of the output signal calculation device of the sensitive element, containing the double valid signal. It completely has no such measurement errors caused by the effects of vertical acceleration and instrumental errors.

\section{Simulation of the operation of the sensing element}

In [10], a mathematical model of a three-coordinate sensitive element of the weapon stabilization informationmeasuring system is presented. Software for its performance modeling under the influence of external perturbations is developed (Fig. 6).

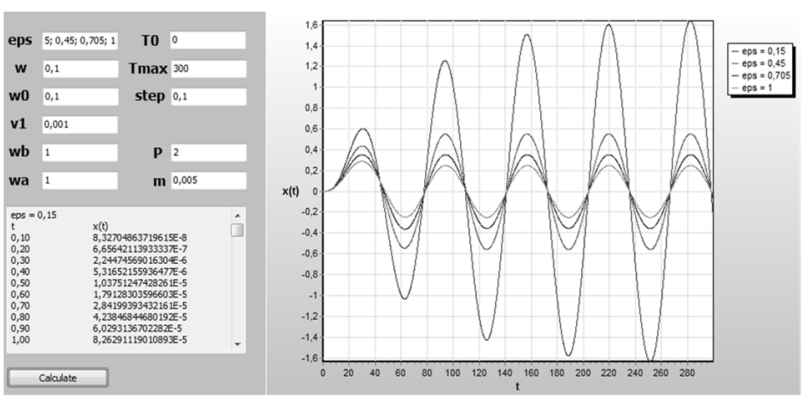

Fig. 6. Interface of the program for developing the sensitive element performance under the influence of the external perturbations.

Graphs of the PG output signal change for different perturbation frequency $\omega$, the damping coefficient $\xi$ and the amplitudes of vibrational acceleration perturbation are obtained. In the result of the performed digital simulation analysis, it is established that (Fig. 7-8):

- when the disturbance frequency is equal to the natural frequency of the sensitive element, the main resonance happens disappearing at $\xi=0.705$;

- at perturbation frequencies less than the natural frequency of the sensitive element, the output signal is not distorted (subharmonic oscillations are established);

- at perturbation frequencies higher than the natural frequency of the sensitive element and $\xi \leq 0,45$ the output signal is distorted (runout happens), that is why it is to the point to increase $\xi$.

\section{Conclusions}

The analysis of the structure and performance principle of the information and measurement system of weapon stabilization, which can be installed on light armored vehicles, is made. The simplest system of indirect stabilization is reviewed; its mathematical description is made. The new precision sensitive element for the information and measurement system of weapon stabilization is suggested. The performance principle of it is introduced. Constructive methods for the new sensitive element measurement accuracy improvement are analyzed. A simulation of the new sensitive element performance under the influence of external perturbations is carried out. 


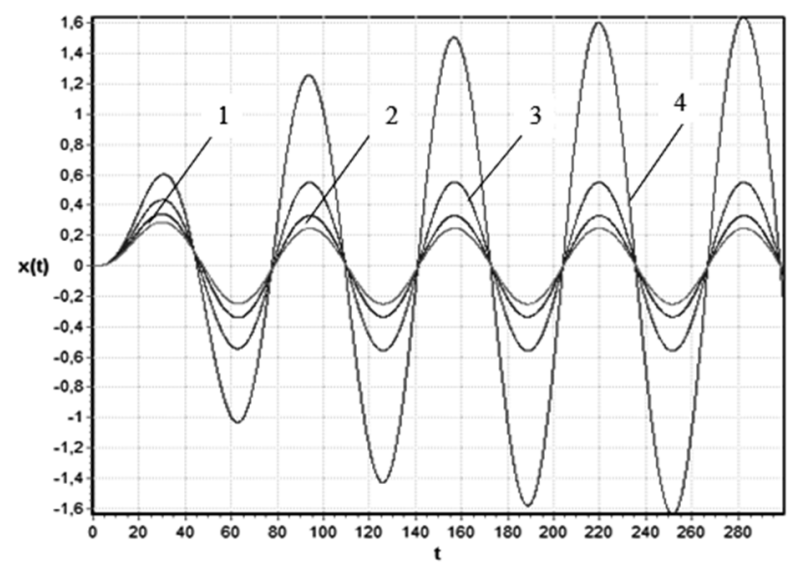

Fig. 7. A typical graph of the amplitude of the output signal $x=x^{\prime}(t), \omega=\omega_{0}=0.1 \mathrm{rad} / \mathrm{s}: 1-\xi=1 ; 2-\xi=0.705$; $3-\xi=0.45 ; 4-\xi=0.15$.

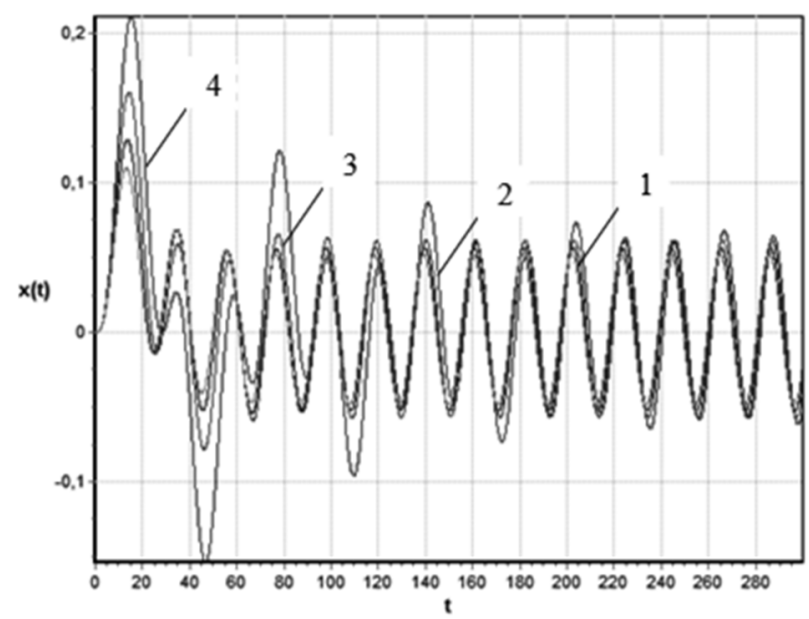

Fig. 8. A typical graph of the amplitude of the output signal $x=x^{\prime}(t), \omega=3 \omega_{0}=0.3 \mathrm{rad} / \mathrm{s}: 1-\xi=1 ; 2-\xi=0.705$; $3-\xi=0.45 ; 4-\xi=0.15$

\section{References}

1. Weapon stabilization systems (2020). http://kza.com.ua/index.php?option=com_content\& view $=$ article\&id $=98 \&$ Itemid $=61$. Accessed $06 \mathrm{Mar}$ 2020

2. A. Tarasenko, Bronetankovaya tehnika Ukrainyi: itogi, potentsial, perspektivyi (Armored vehicles of Ukraine: conclusions, capacity, perspectives). Armored vehicles of Ukraine 4, 29-35 (2008), http://militaryarticle.ru/tekhnika-ivooruzhenie/2008/11678-bronetankovaja-tehnikaukrainy-2. Accessed 06 Mar 2020

3. B.I. Kuznetsova, T.Y. Vasyltsev, O.O. Vaerfolomiev, Sistema navedennya i stabilizaciyi ozbroyennya legkobronovanih mashin $\mathrm{z}$ nejromerezhevim regulyatorom (The navigation and stabilization system of light armored vehicles weaponization with the neural network regulator). Armament system and military vehicles 1(13), 112$116(2010)$
4. V.V. Chikovani, Influence of shock on the vibration amplitude stabilization system of Coriolis vibratory gyroscope resonator. Electronics and control systems 4(34), 56-63 (2012). doi:10.18372/19905548.34 .5717

5. V. Karachun, V. Melnick, I. Korobiichuk, M. Nowicki, R. Szewczyk, S. Kobzar, The additional error of inertial sensor induced by hypersonic flight condition. Sensors 16(3), 299 (2016). doi:10.3390/s16030299

6. K.V. Bazilo, Shemotehnichne modelyuvannya p'ezoelektrichnogo peretvoryuvacha $\mathrm{z}$ dodatkovimi kolivalnimi konturami (Schematic technical modelling of piezoelectric transducer with the additional oscillation contours). National Khmelnytskyi University Gazette 6, 166-169 (2013)

7. E. Shimane, S. Matsumoto, T. Moriguchi, Y. Iwai, R. Uchino, The Study on Performance of MEMS IMU for Launch Vehicle under High Vibration Environment. Transactions of the Japan society for aeronautical and space sciences, aerospace technology Japan 17(4), 421-426 (2019). doi:10.2322/tastj.17.421

8. I. Korobiichuk, Mathematical model of precision sensor for an automatic weapons stabilizer system. Measurement. Journal of the International Measurement Confederation 89, 151-158 (2016). doi:10.1016/j.measurement.2016.04.017

9. A.V. Koval', Simulation of gravimetric measurements by gyroscopic integrator of linear accelerations. Gyroscopy and Navigation 6(1), 344347 (2015). doi:10.1134/S2075108715040070

10. O. Bezvesilna, A. Tkachuk, T. Khylchenko, S. Nechai, Simulation of influence of perturbation parameters on the new dual-channel capacitive MEMS gravimeter performance. Eastern-European Journal of Eenterprise Technologies 6/7(84), 50-57 (2016). doi:10.15587/1729-4061.2016.85463

11. V. Sharapov, Z. Sotula, L. Kunickaya, Piezo-Electric Electro-Acoustic Transducers (Springer, Cham, 2014). doi:10.1007/978-3-319-01198-1 